\title{
Enhanced Recovery After Surgery is Feasible in Laparoscopic Low Anterior Resection for Rectal Cancer
}

\author{
Jeong Seon Jo, M.D., Soo Young Lee, M.D., Hun Jin Kim, M.D., Chang Hyun Kim, M.D., \\ Young Jin Kim, M.D., Ph.D., Hyeong Rok Kim, M.D., Ph.D.
}

Department of Surgery, Chonnam National University Hwasun Hospital, Hwasun, Korea

\begin{abstract}
Purpose: The aim of this study was to compare short-term outcomes in patients who underwent laparoscopic assisted low anterior resection for colorectal cancer. The patients received either conventional perioperative care or the Enhanced Recovery After Surgery (ERAS) procedural care.

Materials and Methods: A retrospective review was conducted in patients who underwent elective laparoscopic low anterior resection for colorectal cancer between May, 2011 and December, 2013. Patients were grouped and analyzed according to the perioperative care program of ERAS and conventional care.

Results: A total of 81 patients received care via the ERAS pathway and 230 patients received care via conventional pathway. There was no significant difference in postoperative morbidity rates $(P=0.381)$. The post-operative morbidity rates were $30.9 \%$ and $25.2 \%$ in the ERAS and conventional groups. No significant difference in hospital stay $(9.0 \pm 6.8$ vs. $8.6 \pm 3.5$ days; $P=0.575$ ) was observed between the 2 groups.

Conclusion: No short-term perioperative disadvantages were found for the ERAS program compared with the conventional perioperative care for colorectal cancer patients who underwent laparoscopic low anterior resection. (Surg Metab Nutr 2015;6:11-15)
\end{abstract}

Key Words: Enhanced recovery after surgery, Laparoscopic low anterior resection

\section{INTRODUCTION}

Surgical outcome of colorectal cancer treatment depend not only on good surgery and tumor biology, but also on optimal perioperative care. Colorectal resections are associated with an in hospital stay of 6 to 11 days and a complication rate of $15 \%$ to $20 \%$. The Enhanced Recovery After Surgery (ERAS) program was developed to improve perioperative care in these patients.[1-3]

ERAS is a multimodal, periopeative management, and care pathway that aims to reduce the stress response to

Received February 17, 2015. Accepted May 28, 2015.

Correspondence to: Hyeong Rok Kim, Department of Surgery, Chonnam National University Hwasun Hospital and Medical School, 322 Seoyang-ro, Hwasun-eup, Hwasun-gun, Hwasun 519-763, Korea

Tel: +82-61-379-7643, Fax: +82-61-379-7661

E-mail:drkhr@chonnam.ac.kr surgery and optimize postoperative recovery.[4] ERAS was associated with improved short-term outcomes that included reduced length of stay, reduced morbidity, faster return of bowel function, earlier mobilization, and lower pain score.[5-10]

Minimally invasive surgery (MIS) has demonstrated superiority in recovery and complications rates, without affecting oncological results or overall and disease free survival.[11] There are reports from smaller study populations of decreased hospital stay with MIS rectal surgery managed with ERAS programs.[12] Therefore, the feasibility of ERAS programs in rectal MIS procedures, especially laparoscopic low anterior resection, needs further evaluation.

The aim of the present study was to evaluate the clinical relevance of the improved perioperative care by 
comparing the mortality, morbidity, and hospital stay in patients who underwent laparoscopic assisted low anterior resection for colorectal cancer and received eihter conventional or ERAS perioperative care.

\section{MATERIALS AND METHODS}

We retrospectively reviewed patients who underwent elective laparoscopic low anterior resection for colorectal cancer by 2 operators in our institution between May, 2011 and December, 2013. A total of 342 patients were identified. Patients with diverting ileostomy, open conversion, and abdominoperineal resection were excluded from the analysis. Because a diverting stoma has reduced morbidity for patients undergoing colorectal surgery to colorectal cancer,[13,14] we excluded patients undergoing diverting stoma in order to prevent its possible bias on morbidity. A total of 311 patients were analyzed.

Patients included in the study were treated perioperatively with either ERAS or the conventional pathway in defined order (Table 1). Patients received different perioperative care of conventional and ERAS programs according to an operator. An enhanced recovery protocol included early oral feeding, active ambulation, and early removal of urinary catheter and drainage tube. Thromboprophylaxis was performed with enoxaparin (Clexane 20 mg s.c.) for 3 days. TNM stage was defined after microscopic examination by pathologists according to the $6^{\text {th }}$ edition of the American Joint Commission on Cancer (AJCC) TNM staging system. The length of stay defined as the time between the date of surgery and date of dis- charge, was the primary end point. Discharge criteria include adequate pain control with or without oral analgesics, no nausea and tolerating to regular hospital diet, no clinical or radiologic sign of postoperative ileus, mobilization and self support as comparable to the preoperative level, and agreement of the patient above all. These discharge criteria were used in both pathways. Secondary outcomes were to evaluate postoperative morbidities including ileus, anastomotic leakage, wound infection, urinary retention, acute kidney injury, and pneumonia. Each morbidity was defined: ileus; No flatus, abdominal distension, nausea or vomiting which prevented oral intake or required therapeutic use of nasogastric tube; anastomotic leak, discharge of bowel content via the drain, wound, or abnormal orifice; wound infection, documented erythema, discharge requiring antibiotics treatment or wound dehiscence requiring closure; unrinary retension, failure to pass urine requiring insertion of urinary catheter; pneumonia, production of $\mathrm{pu}^{-}$ rulent sputum with positive bacteriological cultures, with or without chest radiography changes or pyrexia or consolidation seen on chest radiograph; acute kidney injury, decreased urine production, quantified as less than 400 $\mathrm{ml} /$ day; and fluid and electrolyte imbalance.

Statistical analyses were performed with SPSS ${ }^{\circledR}$ version 21.0 (SPSS, Inc., Chicago IL) for Windows ${ }^{\circledR}$. Analysis of the categorical variables was performed by chi-square test, and analysis of continuous variables was performed by student's $t$-test in order to investigate the differences between the 2 groups. Continuous values were expressed as the mean \pm standard deviation. A value of $\mathrm{P}<0.05$ was

Table 1. Clinical pathway of laparoscopic low anterior resections

\begin{tabular}{lll}
\hline & \multicolumn{1}{c}{ ERAS group } & \multicolumn{1}{c}{ Conventional group } \\
\hline OP day & LMWH, compression stocking, warm blanket, antiemetics & Compression stocking, warm blanket, antiemetics \\
POD 1 & LMWH, water feeding, soft diet, fluid; $1000 \mathrm{ml}$ & $\mathrm{NPO}$, fluid; $3000 \mathrm{ml}$, \\
POD 2 & LMWH, soft diet, foley catheter removal fluid; $1000 \mathrm{ml}$ & Water feeding, foley catheter removal fluid; $2000 \mathrm{ml}$ \\
POD 3 & Soft diet & Soft diet \\
POD 4 & RHD, drain removal & Soft diet, drain removal \\
POD 5 & RHD & RHD \\
POD 6 & RHD & Discharge \\
POD 7 & Discharge & \\
\hline
\end{tabular}

$\mathrm{POD}=$ postoperative days; $\mathrm{OP}=$ operation day; $\mathrm{LMWH}=$ low-molecular weight heparin; $\mathrm{NPO}=$ nothing per oral; $\mathrm{RHD}=$ regular hospital diet. 
considered statistically significant.

\section{RESULTS}

A total of 81 patients were cared for via the ERAS pathway, and 230 patients were care for via the conventional pathway. The mean age was $63.1 \pm 11.0$ years (range, 34 $83)$ in the ERAS group, and $65.4 \pm 11.3$ years (range, 35 83) in the conventional group. There was no significant difference between groups with regard to age, sex, ASA, stage, underlying diseases, and BMI. However, there was a difference in tumor level $(\mathrm{P}=0.018)$ between the 2 groups (Table 2).

The ERAS group had an earlier removal of the drain catheter $(4.3 \pm 2.2$ vs. $5.5 \pm 1.8$ days; $\mathrm{P}<0.0001)$ and foley catheter $(2.6 \pm 1.5$ vs. $3.2 \pm 1.2$ days; $\mathrm{P}=0.001)$ than the conventional group. There was no significant difference in hospital stay $(9.0 \pm 6.8$ vs. $8.6 \pm 3.5$ days; $\mathrm{P}=0.575)$ be- tween 2 groups (Table 3). One patient from the ERAS group died due to myocardial infarction. A reoperation was performed on 6 patients from the ERAS group, and 19 patients from the conventional group.

The post-operative morbidity rates in the ERAS and conventional groups were $30.9 \%$ and $25.2 \%$, respectively. There was no significant difference in the postoperative morbidity rates $(\mathrm{P}=0.381)$ (Table 4). Postoperative ileus occurred in 6 patients from the ERAS group, and $15 \mathrm{pa}^{-}$ tients from the conventional group. These patients were improved by conservative treatments. Anastomotic leakage occurred in 5 patients from the ERAS group, and 18 patients from the conventional group. Reoperation was performed in all patients with anastomotic leakage.

\section{DISCUSSION}

Our study results suggested that ERAS program was as-

Table 2. Baseline characteristics of patients according to the treatment arms

\begin{tabular}{|c|c|c|c|}
\hline Variables & ERAS group $(n=81)$ & Conventional group $(n=230)$ & P-value \\
\hline Age, mean $\pm S D$ & $63.1 \pm 11.0$ & $65.4 \pm 11.3$ & 0.198 \\
\hline Sex, n (\%) & & & 0.266 \\
\hline Male & $60(74)$ & $153(66)$ & \\
\hline Female & $21(26)$ & 77 (34) & \\
\hline $\mathrm{BMI}$, mean $\pm \mathrm{SD}$ & $23.4 \pm 3.1$ & $23.8 \pm 4.8$ & 0.393 \\
\hline Underlying disease, $\mathrm{n}(\%)$ & & & 0.700 \\
\hline Hypertension & $34(42)$ & $94(41)$ & \\
\hline DM & $22(27)$ & $50(22)$ & \\
\hline Pulmonary disease & $2(2.5)$ & $11(4.8)$ & \\
\hline Liver disease & $2(2.5)$ & $6(2.6)$ & \\
\hline Tumor level, n (\%) & & & 0.018 \\
\hline Upper rectum & $57(70)$ & $131(57)$ & \\
\hline Mid rectum & $17(21)$ & $87(38)$ & \\
\hline Lower rectum & $7(9)$ & $12(5)$ & \\
\hline ASA score, $\mathrm{n}(\%)$ & & & 0.641 \\
\hline 1 & $19(20)$ & $45(20)$ & \\
\hline 2 & $57(76)$ & $174(75)$ & \\
\hline 3 & $5(4)$ & $11(5)$ & \\
\hline Operation type, n (\%) & & & 0.001 \\
\hline Low anterior resection & 77 (95) & $230(100)$ & \\
\hline Intersphincteric resection & $4(5)$ & 0 & \\
\hline Operation time (min), mean \pm SD & $149.3 \pm 42.6$ & $146.3 \pm 29.6$ & 0.557 \\
\hline Stage $(A J C C), n(\%)$ & & & 0.283 \\
\hline 1 & $29(36)$ & $77(33.5)$ & \\
\hline 2 & $16(20)$ & $67(29)$ & \\
\hline 3 & $30(37)$ & $77(33.5)$ & \\
\hline 4 & $6(7)$ & $9(4)$ & \\
\hline Neoadjuvant chemotherapy, n (\%) & & & 0.215 \\
\hline Yes & $12(15)$ & $22(10)$ & \\
\hline No & $69(85)$ & $208(90)$ & \\
\hline
\end{tabular}

$\mathrm{SD}=$ standard deviation; BMI = body mass index; $\mathrm{ASA}=$ American Society of Anesthesiologists; AJCC = American Joint Committee on Cancer. 
Table 3. Summary of clinical results according to the treatment arms

\begin{tabular}{lccc}
\hline \multicolumn{1}{c}{ Variables } & ERAS group $(\mathrm{n}=81)$ & Conventional group $(\mathrm{n}=230)$ & P-value \\
\hline Day to passage of flatus, mean \pm SD & $2.1 \pm 1.4$ & $2.37 \pm 1.5$ & 0.114 \\
Day of drain removal, mean \pm SD & $4.3 \pm 2.2$ & $5.5 \pm 1.8$ & $<0.0001$ \\
Day of foley catheter removal, mean \pm SD & $2.6 \pm 1.5$ & $3.2 \pm 1.2$ & 0.001 \\
Day of defecation, mean \pm SD & $4.1 \pm 1.7$ & $4.7 \pm 1.9$ & 0.011 \\
Hospital stay, mean \pm SD & $9.0 \pm 6.8$ & $8.6 \pm 3.5$ & 0.575 \\
Reoperation, $\mathrm{n}(\%)$ & $6(7.4)$ & $19(8.3)$ & 0.808 \\
Readmission within 30 days, $\mathrm{n}(\%)$ & $2(2.5)$ & $5(2.2)$ & 0.878 \\
Death within 30 days, $\mathrm{n}(\%)$ & $1(1.2)$ & $0(0)$ & 0.260 \\
\hline
\end{tabular}

Table 4. Postoperative Morbidities according to the treatment arms

\begin{tabular}{lccc}
\hline \multicolumn{1}{c}{ Complication classification } & ERAS group $(\mathrm{n}=81)$ & Conventional group $(\mathrm{n}=230)$ \\
\hline Any complication, $\mathrm{n}(\%)$ & $25(30.9)$ & $34(25.2)$ & $15(6.5)$ \\
lleus, n (\%) & $6(7.4)$ & $2(0.9)$ & 0.381 \\
AKI, n (\%) & $2(2.5)$ & $18(7.8)$ & 0.799 \\
Anastomotic leak, n (\%) & $5(6.2)$ & $17(7.4)$ & 0.279 \\
Wound infection, n (\%) & $7(8.6)$ & $14(6.1)$ & 0.806 \\
Urinary retension, n (\%) & $5(6.3)$ & $1(0.4)$ & 0.809 \\
Pneumonia, n (\%) & $1(1.2)$ & 0.958 & 0.454 \\
\hline
\end{tabular}

sociated with no increased complications in comparison with a conventional program.

A meta-analysis of 13 randomized controlled trials comparing ERAS and conventional care for various colorectal operations including colorectal cancer surgery revealed that patients under the care of ERAS had a shorter hospital stay by approximately 2.4 days.[15] ERAS shortened the length of hospital stay (LOS) in patients undergoing laparoscopic colorectal resection for colorectal cancer.[16-18] Our study on the other hand, showed that there was no difference in hospital stay $(9.0 \pm 6.8$ vs. $8.6 \pm 3.5$ days; $\mathrm{P}=0.575$ ) between 2 groups. We attributed our results to the lack of strict discharge criteria of our protocol. The optimal outcomes of ERAS were largely dependent on the compliance and adherence to the protocol.[19,20]

The discharge of patients was delayed due to personal reasons of economic circumstances, schedules, and preferences. Furthermore, operators did not strictly keep a discharge criteria. The delayed discharge of patients could affect the readmission within 30 days. Previous study reported that readmission rates in the ERAS and conventional group were $7.7 \%$ and $13.8 \%$, respectively.[21] We found that readmission rates in the ERAS and conven- tional group in our study were $2.5 \%$ and $2.2 \%$, respectively.

It is likely that the combination of elements in ERAS favored an uncomplicated outcome after laparoscopic low anterior resection for colorectal surgery. There was no difference in terms of overall morbidity, reoperation rate, and mortality between the 2 groups. Recent studies also reported that there were no significant differences in postoperative complication rates between the 2 groups. [21,22]

The advantages of ERAS have been widely reported in various operations for colorectal diseases. ERAS is known to provide increased benefits to patients during the postoperative recovery period. However, the impact of such a program on the long-term oncological outcome of colorectal cancer surgery is lacking. Emerging evidence indicates that the application of ERAS to colon cancer surgery effectively inhibits the release of post-operative inflammatory cytokines and mediators, reduces perioperative stress and preserves the post-operative immune system.[23] Better preservation of host immunity is possibly related to greater anti-tumor activity and increased survival in advanced colon cancer.[24] Therefore, long-term studies on such a program in colorectal cancer are needed. ERAS has likely oncological benefit to colorectal 
cancer patients, since it enhances patient's recovery, maintains integrity of the postoperative immune system, increases feasibility of postoperative chemotherapy, and shortens the time interval from surgery to chemotherapy.

There are several limitation in this study. First, this is an observational retrospective cohort study and as such suffers from the unavoidable selection biases. For example, each treatment arms were neither randomly assigned nor surgeons who participating study used both protocol. Second, the sample size is not enough to fully address some issues. Especially, the outcome of anastomotic leak after adopting ERAS could not defined clearly due to this limitation. We hope that future studies should be performed to elucidate this issue with more detail.

In conclusion, the ERAS program for patients who underwent laparoscopic low anterior resection was associated with no increased postoperative morbidities in comparison with a conventional program. The ERAS program was thus feasible for colorectal cancer patients who underwent laparoscopic low anterior resection.

\section{REFERENCES}

1. Zargar-Shoshtari K, Hill AG. Optimization of perioperative care for colonic surgery: a review of the evidence. ANZ J Surg 2008;78:13-23.

2. Fearon KC, Ljungqvist $O$, Von Meyenfeldt $M$, Revhaug $A$, Dejong $\mathrm{CH}$, Lassen $\mathrm{K}$, et al. Enhanced recovery after surgery: a consensus review of clinical care for patients undergoing colonic resection. Clin Nutr 2005;24:466-77.

3. Wind J, Polle SW, Fung Kon Jin PH, Dejong $\mathrm{CH}$, von Meyenfeldt MF, Ubbink DT, et al; Laparoscopy and/or Fast Track Multimodal Management Versus Standard Care (LAFA) Study Group; Enhanced Recovery after Surgery (ERAS) Group. Systematic review of enhanced recovery programmes in colonic surgery. $\mathrm{Br} J$ Surg 2006;93:800-9.

4. Kehlet $\mathrm{H}$. Multimodal approach to control postoperative pathophysiology and rehabilitation. Br J Anaesth 1997;78:606-17.

5. Anderson AD, McNaught CE, MacFie J, Tring I, Barker P, Mitchell C). Randomized clinical trial of multimodal optimization and standard perioperative surgical care. Br J Surg 2003;90:1497-504.

6. Basse L, Hjort Jakobsen D, Billesbølle P, Werner M, Kehlet H. A clinical pathway to accelerate recovery after colonic resection. Ann Surg 2000;232:51-7.

7. Eskicioglu C, Forbes SS, Aarts MA, Okrainec A, McLeod RS. Enhanced recovery after surgery (ERAS) programs for patients having colorectal surgery: a meta-analysis of randomized trials. J Gastrointest Surg 2009;13:2321-9.

8. King PM, Blazeby JM, Ewings P, Longman RJ, Kipling RM, Franks PJ, et al. The influence of an enhanced recovery programme on clinical outcomes, costs and quality of life after surgery for colorectal cancer. Colorectal Dis 2006;8:506-13.

9. Lassen K, Soop M, Nygren J, Cox PB, Hendry PO, Spies C, et al; Enhanced Recovery After Surgery (ERAS) Group. Consensus review of optimal perioperative care in colorectal surgery: Enhanced Recovery After Surgery (ERAS) Group recommendations. Arch Surg 2009;144:961-9.

10. Walter CJ, Collin J, Dumville JC, Drew PJ, Monson JR. Enhanced recovery in colorectal resections: a systematic review and meta-analysis. Colorectal Dis 2009;11:344-53.

11. Laparoscopically assisted colectomy is as safe and effective as open colectomy in people with colon cancer Abstracted from: Nelson H, Sargent D, Wieand HS, et al; for the Clinical Outcomes of Surgical Therapy Study Group. A comparison of laparoscopically assisted and open colectomy for colon cancer. N Engl J Med 2004; 350: 2050-9. Cancer Treat Rev 2004;30: 707-9.

12. Khoo CK, Vickery CJ, Forsyth N, Vinall NS, Eyre-Brook IA. A prospective randomized controlled trial of multimodal perioperative management protocol in patients undergoing elective colorectal resection for cancer. Ann Surg 2007;245:867-72.

13. Bax TW, McNevin MS. The value of diverting loop ileostomy on the high-risk colon and rectal anastomosis. Am J Surg 2007;193:585-7; discussion 587-8.

14. Karahasanoglu T, Hamzaoglu I, Baca B, Aytac E, Erenler I, Erdamar S. Evaluation of diverting ileostomy in laparoscopic low anterior resection for rectal cancer. Asian J Surg 2011;34: 63-8.

15. Zhuang CL, Ye XZ, Zhang XD, Chen BC, Yu Z. Enhanced recovery after surgery programs versus traditional care for colorectal surgery: a meta-analysis of randomized controlled trials. Dis Colon Rectum 2013;56:667-78.

16. Vlug MS, Wind J, Hollmann MW, Ubbink DT, Cense HA, Engel $A F$, et al; LAFA study group. Laparoscopy in combination with fast track multimodal management is the best perioperative strategy in patients undergoing colonic surgery: a randomized clinical trial (LAFA-study). Ann Surg 2011;254:868-75.

17. Haverkamp MP, de Roos MA, Ong KH. The ERAS protocol reduces the length of stay after laparoscopic colectomies. Surg Endosc 2012;26:361-7.

18. Huibers CJ, de Roos MA, Ong $\mathrm{KH}$. The effect of the introduction of the ERAS protocol in laparoscopic total mesorectal excision for rectal cancer. Int J Colorectal Dis 2012; $27: 751-7$

19. Gustafsson UO, Hausel J, Thorell A, Ljungqvist O, Soop M, Nygren J; Enhanced Recovery After Surgery Study Group. Adherence to the enhanced recovery after surgery protocol and outcomes after colorectal cancer surgery. Arch Surg 2011;146: 571-7.

20. Cakir H, van Stijn MF, Lopes Cardozo AM, Langenhorst BL, Schreurs WH, van der Ploeg TJ, et al. Adherence to Enhanced Recovery After Surgery and length of stay after colonic resection. Colorectal Dis 2013;15:1019-25.

21. Khreiss W, Huebner M, Cima RR, Dozois ER, Chua HK, Pemberton $\mathrm{JH}$, et al. Improving conventional recovery with enhanced recovery in minimally invasive surgery for rectal cancer. Dis Colon Rectum 2014;57:557-63.

22. Gustafsson UO, Tiefenthal M, Thorell A, Ljungqvist O, Nygrens J. Laparoscopic-assisted and open high anterior resection within an ERAS protocol. World J Surg 2012;36:1154-61.

23. Wang G, Jiang Z, Zhao K, Li G, Liu F, Pan H, et al. Immunologic response after laparoscopic colon cancer operation within an enhanced recovery program. J Gastrointest Surg 2012;16 :1379-88.

24. Karanika S, Karantanos T, Theodoropoulos GE. Immune response after laparoscopic colectomy for cancer: a review. Gastroenterol Rep (Oxf) 2013;1:85-94. 